\title{
Prefrontal cortex and neurological impairments of active thought
}

\section{Tim Shallice $^{1}$ and Lisa Cipolotti ${ }^{2}$}

1. Institute of Cognitive Neuroscience, University College London, Gower Street, London WC1E 6BT, UK and SISSA, Trieste, Italy. email: t.shallice@ucl.ac.uk

2. Neuropsychology Department, National Hospital for Neurology and Neurosurgery, Queen Square, London WC1, UK and Dipartimento di Psicologia, Universita di Palermo, Italy. email: 1.cipolotti@ucl.ac.uk

Short title: Impairments of active thought

Corresponding Author: Tim Shallice, Institute of Cognitive Neuroscience, University College London, Gower Street, London WC1E 6BT, UK. email: t.shallice@ucl.ac.uk 


\title{
Table of Contents
}

\section{INTRODUCTION}

Overall perspective

Why neuropsychology?

The neuropsychological approach to frontal functions.

\section{BROAD BRUSH ASPECTS OF ACTIVE THOUGHT}

Dual system brain-based models of cognitive control

Active thought and intelligence

Active thought and language

\section{ESSENTIAL PREREQUISITES FOR ACTIVE THOUGHT}

Volition and concentration

Set-Switching and response inhibition

Active monitoring and checking

Working memory

FORMS OF ACTIVE THOUGHT

\author{
Abstraction \\ Deduction \\ Reasoning in well-structured and less well-structured problem-spaces \\ From lateral transformations to strategy shifts \\ Planning for future action \\ THEORETICAL CONCLUSIONS
}




\title{
Keywords
}

Active thought, neuropsychology, prefrontal cortex, supervisory system, reasoning, lateralisation of function

\begin{abstract}
The paper reviews the effects of lesions to the frontal cortex in so far as they impact on the ability to carry out active thought, namely to reason, think flexibly, produce strategies and formulate and realise plans. How relevant neuropsychological studies should be carried out and why are discussed. The relation between active thought and each of intelligence and language are considered. The following basic processes necessary for effective active thought are reviewed: concentration, set-switching, inhibiting potentiated responses and monitoring and checking. Different forms of active thought are then addressed: abstraction, deduction, reasoning in well-structured and ill-structured problem spaces, novel strategy generation and planning. It is concluded that neuropsychological findings are valuable for providing information on systems rather than networks, and especially concerning prefrontal lateralisation of function. A synthesis is presented of the respective roles of the left and right lateral prefrontal cortex in active thought.
\end{abstract}




\section{INTRODUCTION}

\section{Overall Perspective}

This paper is concerned with what neuropsychological findings can tell us about the cognitive processes underlying active thinking. By active thinking we refer to mental processes that allow us to confront situations where we do not respond routinely to the environment but rather we effectively address problems which can be big or small. Active thinking entails a set of complex mental processes for example, those involved in abstraction, deduction and other forms of reasoning between alternative possibilities, switching lines of thought, strategy selection, inhibition of obvious responses and formulating and realising plans. For example, organizing a dinner party would entail many active thinking processes whereas day-dreaming or implicit processes like priming would not be considered active thinking.

A number of well-known neuropsychological tests designed to assess prefrontal function require active thinking. Typical examples are tests such as Wisconsin Card-Sorting (switching lines of thought), Proverb Interpretation (abstraction), Stroop (inhibition) Tower of Hanoi (planning) and tests of fluid intelligence such as Progressive Matrices or Cattell Culture Fair (reasoning between alternative possibilities). Following frontal lobe lesions, performance on these tests is typically impaired. This suggests that the frontal lobes are critically involved in active thinking.

Impairments in active thinking are also exemplified by a number of frontal lobe syndromes which involve release of irrelevant environmentally triggered actions. Examples include the grasp reflex, where the patient is repeatedly instructed not to grasp the doctor's finger as the patient's palm is stroked, and yet does so (De Renzi \& Barbieri, 1992) or the somewhat 
analogous situation where the patient should not move their eyes to a distracting light but does so (Paus et al. 1991). At a higher level, there is utilisation behavior, originally described by Lhermitte (1983). In this case the patient makes a standard afforded action to one of the objects surrounding him, such as dealing from a pack of cards, without being told to do so, or in the so-called incidental form, when explicitly told to do something else (Shallice et al, 1989). These examples highlight behaviors that occur when active thinking processes are absent or impaired through brain injury. Interestingly, these syndromes have been most frequently described in patients with lesions involving medial frontal areas (see De Renzi \& Barbieri, 1992).

Prefrontal functions are involved in many different cognitive domains. They have been well reviewed fairly recently by Sczepanski \& Knight (2014). This paper will therefore focus only on those cognitive domains we consider critical for active thinking. Thus we will discuss individual cognition rather than social and on-going reasoning rather than (long-term) memory, learning, motivation and emotion.

Our paper is structured in the following fashion. The Introductory section will consider why neuropsychological evidence is chosen for review of the cognitive processes underlying active thinking, out of the many cognitive neuroscience techniques available. We then address the methodological approaches adopted for the neuropsychological investigation of prefrontal functions. The second section will briefly outline our theoretical framework for active thinking, which is largely based on the Norman and Shallice (1986) Supervisory System model of prefrontal cortex (PFC) functioning. We will also consider the relationship between active thinking and potentially overlapping cognitive domains such as intelligence and language. The third section will deal with processes that are prerequisites for active thinking, namely concentration, set-shifting, thought inhibition, and monitoring and checking. In the fourth section we will discuss different types of core active thinking 
processes including abstraction, deduction, novel strategy selection, insight, and planning. The final section aims to produce an overall theoretical synthesis.

\section{Why neuropsychology?}

As cognitive processes become more abstract and distant from sensory and motor processes, it becomes increasingly difficult to investigate them adequately using behavioral means alone. Thus, taking theorists working on reasoning about syllogisms, Khemlani \& JohnsonLaird (2012) wrote “...Thirty-five years ago they had only heuristic accounts that explained biases and errors, and so the domain appeared to be an excellent test case for cognitive science. There are now 12 sorts of theories of syllogisms and monadic inferences, and so skeptics may well conclude that cognitive science has failed..." (p.453). A more powerful source of empirical findings seems to be required. Obvious candidates are methodologies related to the brain.

Within human cognitive neuroscience there are two main classes of methodologies. The oldest class are those derived from lesion studies of neurological patients, which have recently been supplemented by TMS, and somewhat more conceptually distant, by the cognitive effects of individual differences in brain structure across the normal population. The second class are those where on-line measures are taken of brain processes while normal subjects carry out tasks - PET, fMRI, EEG, MEG and so on.

If one's aim is to provide accurate anatomical correspondences for known cognitive processes or to provide real-time information on processing, the second class are much to be preferred. Despite this clear advantage of the second class of methodology, the first-class, especially neuropsychology, have complimentary advantages for the development of cognitive theory. This is for at least five reasons: 
(i) If one takes cognitive theory to refer to models like classic box-and-arrow information-processing ones, then neuropsychological data can speak directly to cognitive theory. Appropriate inferences are derived from a set of simple assumptions, first formalised by Caramazza (1986). They are based on the idea of subtraction of components from an overall system. Of course, subtraction in reality is complicated by complex processes related to the recovery process (see Henson et al, 2016 for a good example). However, to a first approximation, subtraction is a plausible characterisation of the effect of a brain lesion. So, this approach was much used in the heyday of cognitive neuropsychology. Moreover, the same set of assumptions can be used to relate such data to connectionist models too (Shallice \& Cooper, 2011). By contrast, methodologies of the second type require complex bridging assumptions, based on physics and physiology, to relate their data to cognitive theory.

(ii) It is generally accepted that activation-based findings do not necessarily imply causal efficacy (see Giliae-Dotan et al, 2015 for a particularly clear example). This possibility is of particular concern for lateralisation of function. Thus, neuropsychological data show language functions to be strongly lateralised. Crossed aphasia is very rare. In a consecutive series of over 1200 aphasics with unilateral lesions, only $4 \%$ had right hemisphere lesions (Croquelois \& Bogousslavsky, 2011). However, neuroimaging studies of language processing often report bilateral activation patterns, if somewhat smaller in size in the right hemisphere (Jung-Beeman, 2005). So, considering merely presence or absence of activation may in effect hide real lateralisation of function. . 
(iii) Neuropsychological data provide additional sources of behavioral evidence that are not generally available from other cognitive neuroscience methods, namely the nature of the responses, in particular, errors made. These can be very informative for specifying the function damaged. We will consider later two examples, of the concrete interpretation of proverbs and of strategy-reflecting responses.

(iv) When carrying out a cognitive neuroscience study on neurologically intact subjects, one is reliant on the theoretical framework of the investigators to set up the experimental question and design. Neurological patients can produce behaviors strikingly challenging theoretical preconceptions. Phineas Gage and HM are the most famous such cases, but there are many others. They facilitate serendipity.

(v) Some problem-solving situations involve a single step change, where the subject makes a single change in strategy. Examples are those involving insight (see section four on Novel strategy selection and insight). They cannot be effectively studied using standard activation-based methods that require summing over multiple trials, since the critical situations cannot be reproduced; a repeat would no longer be novel. Instead damage to the relevant systems may prevent strategy change occurring, hence allowing relevant investigation.

We will therefore address primarily findings from neuropsychological studies, and consider other methodologies where their findings help interpret our principal type. Of course, neuropsychological methods have their own limitations, which we now discuss.

\section{The neuropsychological approach to frontal functions.}

Three main types of method have been used by researchers concerned with making inferences about normal cognitive function from neuropsychological data - the single case 
study (including its close relation - the multiple single case study), the case series and the group study. On the first approach individual patients are selected for study depending on their theoretical interest. In the second and third, all patients who fit the appropriate criteria are reported. On the second approach each patient is treated as a separate test of relevant theories, whilst on the third approach, results are averaged across all patients in a group.

Historically, researchers have tended to favour one approach and reject others. Instead, one of us (Shallice, 2015) has argued that all three approaches are legitimate, but have different potential problems, and so are more powerful in combination. This needs to be qualified, as far as prefrontal functions are concerned. Often performance of paradigms sensitive to prefrontal lesions can have a large range of performance in the normal population - consider as an example the Stroop test. Impairment then becomes more easily detected using group studies, due to variance reduction with increased $n$.

In practice, the anatomically based group study, where the patient is allocated to a group according to their site of lesion, is the most widely used method for studying prefrontal functions. It comes in two forms. In one, the more traditional approach the anatomical regions are decided a priori. In the oldest such version, the classical approach, there is simply a comparison between unilateral left and right frontal lesioned patients. In a more refined approach (the Stuss-Alexander method), frontal patients are divided into left lateral, right lateral, superior medial and inferior medial (including orbital), this division being based partly on statistical grounds and partly on clinical ones (Stuss et al, 1998). In the modified Stuss-Alexander method the two medial groups are combined. 
The alternative approach (the critical lesion localisation method) uses the range of performance produced by patients in a series. It determines whether there are patients with lesions in a particular region who perform worse than those with lesions elsewhere, without specifying the region in advance. A package is used such as Voxel-based Lesion Symptom Mapping (VLSM) - now sometimes called Lesion Behavior Mapping (LBM) (Bates et al, 2003; Rorden \& Karnath, 2004).

Recently, Mah et al (2014) have criticised existing methods of this type, which make the simplifying assumption that damage to any voxel is independent of that to any other voxel. Unfortunately, the assumption is flawed when applied to brain lesions caused by stroke. In this case, the arterial tree structure of the vascular system means that there will be a high correlation between damage to functionally critical and non-critical regions fed by the same artery. Mah et al advocated a high-dimensional multi-variate approach. However, to our knowledge, this has yet to be applied in an analysis of the effects of prefrontal lesions. Related criticisms may apply to brain tumours, but if they do the associated non-critical regions will not be the same. This makes replication, especially across aetiology, very useful. This is also so for a second problem - the existence of large 'silent' regions due to insufficient patients for complete coverage.

In fact, for the purpose of drawing inferences to the separability of executive systems, the precise anatomical location of a critical area is not important. Performance on a given test requires many subprocesses. So, the inferential logic of cognitive neuropsychology depends on the relative performance of the patient across multiple tests. We will adopt an analogous approach using groups. If, the critical areas for one test do not overlap with those of another, we will take this as evidence that the two tests do not rely on the same set of subsystems. We 
will discuss later how two tests that appear to involve inhibition - the Stroop and the Hayling B Sentence Completion Test - lateralise differently in PFC, and therefore that the most critical processes for performing the two tests differ.

Instead, there can be commonality of lesion area in the performance of different tests. This then puts on the intellectual agenda, the possibility that the cognitive resources required by test performance may overlap cognitively as well as anatomically. For example, Tsuchida \& Fellows (2013) used VLSM on the performance of 45 frontal patients on three tests - taskswitching, the Stroop and a spatial search task. The authors found similar left ventrolateral critical regions for the first two tasks. A different more medial region was critical for the spatial search task. The authors held that the existence of a common critical area for the first two tasks meant that "..they are likely to be related to disruption of a single underlying process..” (p.1797). We consider that it provides suggestive evidence only.

Adopting a group study methodology is though beset with a host of methodological problems. Typically, patients differ widely in age and premorbid cognitive abilities. In addition, lesions vary greatly in aetiology and size. Moreover, these two types of factor can interact in a complex fashion. Thus, Cipolotti et al (2016) examined two tests sensitive to prefrontal damage - Advanced Progressive Matrices and Stroop. Increasing age was found to exacerbate the effects of frontal damage, as measured using age-specific norms. This exacerbated age effect on executive performance in frontal patients was not ameliorated by proxies of cognitive reserve such as education or IQ (Macpherson et al, in press). This suggests that any behavioral effect of a lesion can only manifest itself when influenced by many strong confounding factors. Large samples of patients and well-matched subgroups are therefore required. 
How, then is one to proceed in practice? One approach is to limit the sample by, say, restricting selection to a particular type of aetiology such as vascular. Thus patients in subgroups should then be better matched, In support of this view; Karnath \& Steinbach (2011) argue that it is best to restrict patient samples to those suffering strokes and reject other aetiologies in particular, tumours. The authors suggest that the effects of tumours are too diffuse and not well localised. In fact, there are clear examples showing that postoperatively tumours can give strong localisation effects (for a particularly clear example see Papagno et al, 2011). Moreover, if one was to include only patients with vascular lesions, collecting a large sample of frontal patients with well-matched subgroups for a new set of tests would in practice take much too long.

A common practice, therefore, is to mix different aetiologies in the patient sample, in order to obtain a large enough group. But are the effects of strokes and tumours, say, even roughly equivalent when affecting similar parts of cortex? To answer this question, Cipolotti et al (2015a) et al compared 100 frontal patients with four different types of aetiology on four frontal executive tasks (Advanced Progressive Matrices, Stroop Colour-Word Test, Letter Fluency-S; Trail Making Test Part B). The four groups consisted of one vascular group and three with different types of tumour - high-grade gliomas, low-grade gliomas and meningiomas. The groups did not differ significantly in size or location of lesion. Strong behavioral effects were found of age and premorbid cognitive abilities on performance of the frontal tests. However, on only one test - Trail-Making Part B - was a significant difference between aetiologies obtained when age was partialled out in an ANCOVA. Critically, the significance did not survive Bonferroni correction, as there was no reason to consider TrailMaking, which later research shows not to be specific for frontal lesions (Chan et al, 2015), 
to be more susceptible to differences in aetiology than the other three tests. We therefore conclude that it is acceptable practice to mix aetiologies to overcome the great variability in the population under study.

We will therefore include all types of neuropsychological method in our paper, but concentrate on the a priori groups approach. We will note the number of relevant patients as small group size results are especially likely to be biased by the idiosyncracies of a few patients or by imperfect matching across subgroups.

\section{BROAD BRUSH ASPECTS OF ACTIVE THOUGHT}

\section{Dual system brain-based models of cognitive control}

Within the reasoning literature a variety of so-called dual system models have been put forward. Most of them differentiate between a fast, automatic and unconscious mode of processing and a slow deliberate conscious one (Kahneman \& Frederick, 2002). In the reasoning field, the two are often called the products of system 1 and system 2 respectively (Stanovich, 1999).

Before the development of dual system models of reasoning, the Russian neuropsychologist Alexander Luria (1966) had argued that neuropsychological evidence supports a theoretical framework in which prefrontal cortex contains a system for the programming, regulation and verification of activity-adopting the terminology of the reasoning literature, a system 2 . This prefrontal system implements its functioning by calling upon more posterior systems in the cortex - a system 1. A number of neuroscientists have adopted a related type of dual system model framework for conceptualising PFC function in information-processing terms (see, Shallice, 1982, Miller \& Cohen, 2001 and Duncan, 2010). In this article, we will adopt Norman \& Shallice's (1986) framework. 
Contention Scheduling - the system 1 of this framework - is the lower level control system which can effect routine thought and action operations. It operates in production-system fashion including selecting action and thought schemas involving more posterior dedicated processing systems and connections (Cooper \& Shallice, 2000). The syndromes discussed in the Introductory section as examples of non-active thought, such as utilization behavior, represent Contention Scheduling operating in isolation.

If Contention Scheduling cannot cope with a non-routine situation, a second higher-level control system comes into play, the Supervisory System, held to be in prefrontal cortex. The Supervisory System is responsible for the control mechanisms that modulate Contention Scheduling top-down by boosting relevant action and thought schemas to allow novel goal directed behavior. The Supervisory System is loosely equivalent to the Executive System or Control Processes in other theoretical frameworks. Where it differs is being more specific about what it modulates and how. It is the key system involved in active thinking.

Another major brain-based model also descending intellectually from Luria's ideas is the multiple demand network approach of Duncan (2010). Duncan \& Owen (2000a) using neuroimaging found that more difficult tasks in many different domains - such as perception, response selection and working memory - activate the same set of regions, mainly in the frontal and parietal cortices, so-called multiple demand regions. These regions are held to have the function of programming other regions of the brain to carry out non-automatic tasks. This is a similar function to that held to be carried out by a Supervisory System. Duncan (2013) also argues that “...the fMRI literature contains little consensus on clear repeatable functional distinctions" ( $\mathrm{p}$ 41) between different regions within the multiple demand network. We will address how the neuropsychological evidence relates to the two models and to equipotentiality later.

\section{Active thought and intelligence}


Duncan et al (2000) also argued that the multiple demand regions are the seat of fluid intelligence, $g$. Thus they made a major link to another cognitive domain, intelligence, and aim to support $g$ as a solid scientific concept.

The neuropsychological literature does not support the idea that reduced fluid intelligence is a sufficient account of frontal patients' executive impairments. Roca et al (2010) showed it is for some tests (eg. Wisconsin Card-Sorting). However, for others such as the Hayling B , both they and Cipolotti and colleagues (2016) have demonstrated that frontal patients' impairment cannot be fully explained by reduced fluid intelligence. Similarly impairments in other executive tests such as Stroop and Proverb Interpretation were shown not to be accounted by an effect on $g$.

Duncan \& Owen's claim about fluid intelligence was, though, specifically about multiple demand regions. To test that, Woolgar et al (2010) gave the Cattell Culture Fair IQ test to 80 patients with cortical lesions. The volume of lesions in multiple demand regions and outside those regions was assessed. For the group as a whole, there was a significant correlation between the IQ score and MD-volume, and the result remained highly significant when total lesion volume was partialled out. However, for the 44 pure frontal patients, the correlation was no longer significant, if non-MD-volume was partialled out. So, as far as PFC is concerned, the theoretical claim was not strongly supported from neuropsychology.

\section{Active Thought and Language}

Thought and language processes are intertwined in numerous complex ways (Gentner \& Goldin-Meadow, 2003), but in the mature adult brain how independently can active thought take place without language? One potential line of evidence comes from aphasia: can aphasics reason? This has been investigated in quite a number of aphasic patients in whom relatively preserved reasoning has been shown (Varley et al, 2014). However, studies have 
tended to be rather loose, relying on essentially clinical reports or on a fairly crude analysis of the processing problems of the patients. An exception is the study of Varley et al (2005) where three patients with severe problems in comprehension and production of syntax were given a variety of arithmetic and calculation tasks. Two of the patients were near ceiling on some calculation tasks with quasi-syntactic aspects, such as three figure subtraction, including problems with negative answers. They also performed adequately, but not perfectly, on problems involving interpretation of brackets. Thus it appeared that the understanding and execution of syntactic operations could be relatively preserved in arithmetic, when such operations were severely impaired in language.

Grammatical encoding is, however, part of what Levelt (1989) characterised as the Formulator stage of language production. It can be inferred from such studies that the Formulator and Articulator stages operate relatively specifically within the language domain as opposed to the thought domain. The key issue therefore relates to the so-called Conceptualizer stage, which precedes them in language production. It produces what Levelt calls the preverbal message, which on our approach requires active thought. Can, however, active thought occur without the involvement of Conceptualizer stage processes?

A relatively little-known aphasia syndrome bears on this question. This is a subtype of the clinical category of transcortical motor aphasia, called dynamic aphasia, first described by Luria (1970). He described two patients who could answer questions but were incapable of narrative speech. In dynamic aphasia, the inner mechanics of the language system - the Formulator and Articulator stages - appear to operate relatively normally, but the patient says little especially in spontaneous speech. For instance, patient ROH of Costello \& Warrington (1989), when asked to describe his last holiday, produced in 30s only "I'm ...". Typically in 
sentence generation tasks the patient failed to produce a response or was extremely slow. However, some direct questions could be answered appropriately and any sentence that was produced was lexically, syntactically and morphologically correct. What appears to be impaired is the Conceptualizer stage.

About 10 other patients of this type have been described as single cases. A massive influence on the performance of these patients is the range of alternatives that are potentially available to the speaker (Robinson et al, 1998). When this is high, the dynamic aphasia patient typically cannot respond. But, when the situation allows only a very restricted set of possibilities, the patient typically produces a correct sentence. For instance, patient ANG of Robinson et al, was given a range of tasks which involved this contrast. Thus, when she was asked to produce a sentence including a common object such as telephone, which was shown as a picture, she scored 0/6. On the other hand, when presented with a simple scene to describe (e.g a girl ice skating), she scored 34/34. When asked to produce a sentence including a single proper name (e.g. Hitler) she scored 26/28, saying for instance "Hitler is one of those wicked people that should never have been born". But given a single common name (e.g. sea), where the range of alternatives is greater, she scored only $14 / 28$, saying in this case "no idea".

Related results were obtained at the same time by Thompson-Schill et al (1998) using a task in which patients were asked to generate a verb given a noun. Nouns were divided into two groups according to the diversity of responses given by controls. Four patients with posterior left inferior frontal lesions had significantly more difficulty with "high selection" (ie. inconsistent) verbs than with "low selection" verbs, compared with controls. Nine patients with lesions elsewhere in the frontal lobes did not have this problem. 
ANG, too, had a left inferior frontal gyrus lesion. This localisation of the main form of dynamic aphasia is supported by a group study. Robinson et al (2010) found that a subgroup of 12 patients with lesions involving the left inferior frontal gyrus was significantly worse than 35 patients with other frontal lesions and normal controls in generating a sentence from a high-frequency word, but not for low-frequency words where selection requirements would be less.

These findings all fit with the dynamic aphasic patient's inability being one of constructing the preverbal message at the Conceptualiser stage, if this is at all difficult because there are many alternative possibilities. Is this a problem that affects active thought processes in situations where language is not required? Individual dynamic aphasic patients can perform apparently much better on reasoning tasks. For instance, patient $\mathrm{CH}$ (Robinson et al, 2005), a similar dynamic aphasic to ANG, but somewhat less severe, performing in the high average range on the IQ test, Advanced Progressive Matrices and in the superior range on WAIS Block Design. However, such a comparison involves many disparate cognitive components. It is not comparable with the Varley et al study of syntactic aspects of arithmetic, where there was excellent matching between verbal and non-verbal tasks.

One type of task which requires active thought and has been studied in both verbal and nonverbal forms in the same patients is that of fluency - generation of items defined by a particular criterion. Phonemic fluency, generating as many words as possible in a fixed time beginning with a particular letter, has been extensively studied by neuropsychologists since the pioneering work of Brenda Milner. It is standardly much impaired in dynamic aphasic patients. In a study of Robinson et al (2012) performance on this test was compared with that 
on seven other fluency tasks. In a sample of 40 frontal patients, out of the 11 who performed worse than any healthy control on phonemic fluency, 6 had left inferior frontal gyrus lesions, as one would expect if it is a sign of dynamic aphasia.

Patients with left lateral lesions in the sample did not generally have word production impairments. On a naming test their scores were similar to those of right lateral lesion patients and not significantly different from those of normal controls. Yet on phonemic fluency they produced only just over $50 \%$ of the number of words that right lateral patients did. By contrast, on producing as many designs as they could, given certain constraints, they performed equally well. Thus $\mathrm{CH}$, for instance, was well within the normal range. Even more surprising, the left lateral patients performed similarly to the right lateral patients in the ideational fluency task- eg. "How many uses you can think of for a brick?". By comparison with right lateral lesion patients their fluency deficit was restricted to verbal material. We assume that a phonemic fluency deficit, if word production processes are intact, is a sign of impairment in the production of the preverbal message by the Conceptualiser stage. Then, it would appear that this process, at least in part, is purely in the language domain and not just reliant on general active thought processes.

\section{ESSENTIAL PREREQUISITES FOR ACTIVE THOUGHT}

\section{Volition and Concentration}

In this section, we deal with processes which might be considered as the nuts and bolts of active thought. We start with the most basic prerequisites for active thought, volition and concentration. Clinically, syndromes such as apathy and akinetic mutism, the failure to initiate actions or speech (Cummings, 1993), which represent the extreme loss of volition, have been associated with lesions to the medial PFC. 
Formal neuropsychological testing supports the idea of a weakening of processes underlying volition in superior medial prefrontal lesions. In the so-called ROBBIA set of studies (Stuss \& Alexander, 2007; Shallice \& Gillingham, 2012), the Stuss-Alexander subdivision of the frontal cortices with $40+$ frontal patients was adopted. These studies included simple reaction time, two versions of choice reaction time, task switching and go-no-go. In none of these paradigms was the left or right lateral or the inferior medial group significantly slower than the normal controls. In all of them, however, the superior medial group were significantly slower than normal subjects and, in most, significantly slower than the other patient groups. Moreover, the effects were large. Thus, in one task, the healthy control group took on average $607 \mathrm{~ms}$, the other three patient groups from 533 to $643 \mathrm{~ms}$, but the superior medial group $821 \mathrm{~ms}$. In addition, in the more difficult conditions, such as the switch condition in task switching compared with the repeat condition, the superior medial group were disproportionately slowed.

Stuss et al $(1995,2005)$ argued that the primary impairment of the superior medial group in these tasks is one of "energisation". They argued that on the Supervisory System model, contention scheduling operating alone would not be optimal in reaction time tasks. For instance, a selected schema would gradually lose activation over several seconds. Thus, for better performance, top-down boosting of lower-level action schemas would be needed. Energisation, then, is seen as the process required toinitiate Supervisory System operations. This closely corresponds to a number of characterisations of the function of the anterior cingulate derived from functional imaging, such as those of Posner \& Girolamo (1998) and Kerns et al (2004). It may be seen as the material substrate of volition and the basis of concentration.

On this approach, impairments following superior medial lesions should be found much more widely even on cognitively simple tasks. They are. Thus, MacPherson et al (2010) 
investigated the performance of 55 frontal patients, subdivided into medial, orbital and lateral subgroups, on the Elevator Counting subtest (Manly et al., 1994). This assesses the ability to sustain attention by presenting a long series of tones at a slow rate. Optimally one simply counts the tones. Medial and left lateral groups were significantly impaired on the task compared to healthy controls, with the medial group making $13 \%$ errors in comparison to the controls $1.6 \%$. In contrast, the right lateral patients were not impaired.

Energization impairments could also account for certain medial frontal findings reported in two studies discussed above. In Robinson et al's (2012) fluency study, the medial frontal group, unlike the lateral frontal groups, was impaired on all eight fluency tasks so an energisation account is more plausible than a purely cognitive one. Medial frontal lesions are also the predominant site for the grasp reflex and utilization behavior, where the task is simple and all that is required is to realise the will to do it. In lay terms the superior medial region can be seen as the locus of the system producing volition and concentration.

\section{Set-Switching and Response Inhibition}

A second prerequisite for active thought is flexibility. Classically the best-known deficit following prefrontal lesions was, indeed, that of switching set. This leads to a consequent increase in perseveration, as in the Wisconsin Card Sorting test, which loads heavily on the ability to switch responding from one perceptual dimension being critical to another (Milner, 1963).

Such clinical tests are, however, complex and have multiple components including a discovery one. Much cleaner are so-called 'task switching' paradigms in which two simple tasks, which use the same stimuli, are carried out repeatedly in a rapid random ordering. Three studies have used such paradigms with 35 or more frontal patients (Aron et al, 2004;

Shallice et al, 2008; Tsuchida \& Fellows, 2013). All three studies showed left frontal patients 
to have either increased error rates early in learning (Shallice et al, 2008) or increased reaction times each time the task switched. Aron et al suggested (2004) that what is impaired in these patients is top-down (Supervisory) control of task-set (action schema). Regarding the critical anatomical areas, a VLSM analysis carried out by Tsuchida \& Fellows was in agreement with a meta-analysis of functional imaging studies carried out by Derrfuss et al (2005), suggesting that the left inferior frontal junction is critical for task switching.

However, Aron et al also reported increased error rates in task switching in patients with right ventrolateral lesions. They attributed this to impairment in response inhibition. However, no such effect was found in either of the other studies. Aron et al (2003) had previously used a standard response inhibition task from human experimental psychology, namely the stop signal task, with the same 17 right frontal patients, but unfortunately no other frontal group. For five right frontal subregions, the correlation between amount of damage to the subregion and poor performance on the task was examined. For three of the regions the correlation was significant, but for one - the inferior frontal gyrus - it was very high. The authors argued that this was the critical region involved in response inhibition, with the other significant effects arising due to correlations between amount of damage in neighbouring regions.

Very different results were, though, obtained by Picton et al (2007) who studied 43 frontal patients with another response inhibition task - go-no-go. They found the critical areas for false alarms were left areas 6 and 8, areas Aron et al did not investigate. The four patients with lesions there made $30 \%$ false alarms. By comparison, the 13 patients with right ventrolateral lesions made only $12 \%$ false alarms, not significantly different from the control (8\%). Thus, the effects found in Aron et al's right frontal patients did not replicate in the two other task switching studies. The neuropsychological evidence overall fits better with a different perspective coming from neuroimaging suggesting that the role of the right inferior 
PFC in such tasks is bottom-up attention rather than inhibition (Hampshire et al, 2010).

When a stop signal occurs after the initiating stimulus, attention must then be switched to the new stimulus. This is not required in Go-no-go tasks.

\section{Active monitoring and checking}

Error detection is an ubiquitous aspect of human active thought, especially when a new skill is being acquired. It begins with a mismatch between actuality and expectation but this can be detected by a variety of means, some very subtle (Rizzo et al, 1995). So monitoring and checking are basic processes late in the time course of active thought. Neuropsychologically, these processes have long been thought to be controlled by dorsolateral PFC systems (Petrides, 1994).

Neuropsychological evidence suggests they are, at least partly, lateralised to the right. Stuss et al (2005) asked 38 frontal patients, anatomically divided into the four Stuss-Alexander groups, to carry out reaction time tests, when the stimulus was preceded by a warning signal which occurred randomly from $3 \mathrm{~s}$ to $7 \mathrm{~s}$ before. For the simple RT condition, controls responded $30 \mathrm{~ms}$ to $40 \mathrm{~ms}$ more rapidly to the long than the short warning intervals - the socalled foreperiod effect. This is to be expected as the conditional probability of the stimulus occurring in a particular interval increases with the foreperiod. Three of the four frontal patient groups behaved in an identical fashion. One exception was the right lateral group which were actually slower in the long foreperiod condition. By contrast, when the foreperiod was fixed over a block of trials, the right lateral group behaved normally. Stuss et al argued that in the variable foreperiod condition, the right lateral group failed to monitor that no stimulus had occurred and so did not increase preparation. When monitoring was not required because the foreperiod was constant over a block, they behaved normally. Thus active monitoring was held to occur in the right lateral frontal area. 
Qualitatively similar results have been obtained in a TMS study of Vallesi et al (2007a) in which stimulation of right dorsolateral PFC was contrasted with that to left dorsolateral PFC and to right angular gyrus. In a more direct attempt to replicate the precise paradigm used by Stuss et al, Vallesi et al (2007b) studied 58 patients with fairly focal tumours. They obtained a partial replication. Premotor patients and parietal patients had foreperiod effects of the order of 30-55ms both before and after operation, the same as normal controls. Left prefrontal patients showed a reduced foreperiod effect of $15-25 \mathrm{~ms}$, both before and after operation. The right prefrontal patients, however, were completely normal before operation with a foreperiod effect of $55 \mathrm{~ms}$, but this was drastically reduced to $10 \mathrm{~ms}$ after operation.

A number of neuroimaging studies point to a similar conclusion with respect to the involvement of right rather than left PFC. Thus, Fleck et al (2006) found that it was the right lateral PFC, too, which was more active in low confidence judgements where more monitoring is needed than in high confidence ones in both memory and perceptual tasks (see also Sharp et al, 2004: Chua et al, 2006; Yokoyama et al, 2010 and for another neuropsychological example Reverberi et al, 2005, but with only the first and fourth of these giving a specifically lateral localisation within the right PFC). Overall, there is some support for lateral regions within the right PFC being the most critical for active monitoring processes.

\section{Working memory}

The reader may be surprised that an obvious requirement for active thought that has not been mentioned is working memory. Working memory has been associated with the lateral PFC since the neurophysiological work of Fuster and Goldman-Rakic. However, these classic neurophysiological experiments typically involved the monkey holding one position in space 
for up to 30 s or so. Human working memory tasks involve the subject making operations on the much greater contents of a short-term store.

When short-term memory tasks are given to patients with frontal lesions, they can exhibit no deficits if operations do not need to be carried out on the contents of the relevant short term memory store. Thus, D'Esposito \& Postle (1999) reviewed all studies they could find that compared groups of patients with lateral frontal lesions and normal controls on tasks that only load on short-term store capacity and do not involve operations. There were eight such studies for digit span and four for spatial span; none showed a significant difference between the two groups. Thus, while "working memory" tasks can produce deficits in frontal patients, the impairment does not appear to be of storage, but of monitoring or manipulation, as argued by Petrides (1994). We have just discussed monitoring above. In the next section, we will discuss how manipulation can take different forms, each associated with different prefrontal regions.

\section{FORMS OF ACTIVE THOUGHT}

\section{Abstraction}

A key human ability for much higher level thinking is the ability to abstract. Goldstein (1936), having worked with soldiers with war wounds and particularly those affecting frontal cortex, described them as having a 'loss of abstract attitude'. Goldstein's concept 'abstract attitude' was rather complex. However, one way it can be operationalised is with the clinical test of giving the interpretation of proverbs. 46 patients with frontal lesions, subdivided into left lateral, right lateral and medial groups, were tested by Murphy et al (2013) using a Proverb Interpretation test (PIT) adapted from Delis et al. (2001). This assesses the ability to interpret a statement in an abstract rather than a concrete sense. Thus, 
for "Rome was not built in a day", a generalised understanding is that any great achievement takes patience and time to complete. A concrete understanding may refer to the time it takes to complete buildings or infrastructure or even to establish the Roman Empire. Medial frontal patients were the only frontal subgroup significantly impaired on the PIT, relative to healthy controls. However, their most frequent responses were 'partially correct' ones (e.g. "Things take time, but you will get there in the end."; so an energisation deficit seems plausible. However, looking in more detail at the errors made by the left lateral group, $45 \%$ were concrete, indicating an inability to produce an abstraction. By contrast, only $12 \%$ of right lateral errors were and $8 \%$ of those made by healthy controls. A related finding was made by McDonald et al (2008) who found that epileptic patients with a left frontal focus produced poorer abstraction responses on this test than those with a right frontal focus. The left lateral region seems critical for abstraction, at least in the verbal domain.

Neuropsychological studies have not yet produced a tighter localisation of any abstraction process in the comprehension of so-called "figurative language". Imaging studies are not entirely consistent, but the most common site is the left inferior frontal gyrus ( e.g. Rapp et al, 2004; see also Papagno et al, 2009 for convergent TMS evidence). Shallice \& Cooper (2013) have argued that the representation of abstract concepts requires a neural architecture that supports the construction of hierarchical structures and this is carried out in the left inferior frontal gyrus.

Of course, abstraction also occurs in non-verbal domains. For instance, it is an important component process in carrying out non-verbal IQ tests, such as the Progressive Matrices or the Cattell Culture Fair. However, to tackle these tests requires many other processes too, so they cannot easily be used to localise non-verbal abstraction. One study that begins to bear on this issue is that of Reverberi et al (2005). They tested 40 frontal patients on the Brixton task (Burgess \& Shallice, 1996a) where subjects must abstract the rules of how a blue circle 
moves across successive cards, each containing a 2x5 array of circles. Left lateral patients were impaired even with good working memory but this was not the case for the other frontal groups. Recently, Urbanski et al (2016) used analogy tasks which are somewhat simpler than fluid intelligence tests, yet require abstraction. Patients were requested to find an analogy between a source set and one of two candidate sets of coloured letters of varying size. The critical region using VLSM was the anterior lateral PFC, again on the left. However, only 27 patients were included in this study, so coverage of the frontal lobes was rather patchy.

\section{Deduction}

Induction is the process by which one produces a novel conclusion from the information currently available, prototypically in the articulation of a new scientific theory. Producing a novel abstraction, the process just discussed, is a key aspect of induction. The complement to induction within reasoning is deduction, where conclusions follow logically and so certainly from the assumptions, the premises. Deduction is, however, somewhat difficult to isolate neuropsychologically as tests typically involve multiple premises. So, in addition to language comprehension, it relies heavily on working memory. While the effect of this factor can be mitigated by allowing premises to remain visible, it is difficult to eliminate completely.

With functional imaging, the complex stages of processing involved in deduction can be tracked over time. Thus, Reverberi et al (2010) used a clever complex design to attempt to isolate in time when subjects, following interpretation of premises, were making logical inferences. Activation increased particularly in left areas 44 and 45 . This result is broadly consistent with earlier functional imaging studies of deduction (eg Goel et al, 2000). However, the complexity of this study would make converging neuropsychological data valuable. Yet lesions to the putatively critical areas typically produce aphasic problems which interfere with the interpretation of individual premises. Probably one of the most extensive 
study of classical deduction in frontal patients was conducted by Reverberi and colleagues (2009). 36 frontal patients were tested on their ability to process one-, two-, or three-premise syllogisms. However, aphasic patients were excluded and this resulted in no patients having lesions overlapping the critical areas left 44 and 45 . Notably, however the performance of right lateral patients was indistinguishable from healthy controls, unlike that of left lateral and medial patients. Deduction like abstraction is a left frontal process at least when the stimuli used are verbal.

\section{Reasoning in well-structured and less well-structured problem-spaces}

A well-structured problem-space is one, where, as in games like chess or puzzles like the Tower-of-Hanoi, the start position and goal are clearly specified. The consequences of selecting one from the finite set of alternatives available at any stage of problem solution are also well-specified in advance. By contrast a less well-structured problem-space, more typical of real-life, is a problem situation where at least one of these conditions does not hold, as in planning cooking a meal for guests.

Tower tasks involve moving balls on pegs to achieve a goal position in the minimum number of moves. They constitute a non-verbal well-structured domain and have been extensively investigated neuropsychologically. The two studies involving most patients are a Tower of London study of Shallice (1982) with 61 patients and a Tower of Hanoi study of Morris et al (1997) with 59 patients. Both tasks included conflict situation trials in which early in the solution the subject must move a ball in the opposite direction to its eventual goal peg. The two studies used the classical group approach and both found a selective impairment in left frontal patients. Of particular interest, in the Morris et al study this was specifically for conflict situation trials occurring relatively early in the testing period. 
Tasks such as these require what Petrides (1994) called manipulation of working memory contents, which he localised in dorsolateral PFC. In particular, they involve, among other processes, updating of the contents of working memory (Miyake \& Friedman, 2012). These two Tower studies do not speak to the specific localisation within the left frontal lobe. More recently, functional imaging studies of these tasks generally support the Petrides view (Kaller et al, 2011; Crescentini et al, 2012). Thus different processes are presumably involved from those underlying verbal deduction.

Returning to the issue of less well-structured problem spaces, Goel et al (2007) used tasks which were either explicitly spatial or could be mapped onto spatial, such as "Mary is smarter than John. John is smarter than Michael. Mary is smarter than Michael. Does it follow?" For half of the problems it did not follow. For these, half again of the problems were indeterminate like "Sarah is prettier than Heather. Sarah is prettier than Diane. Diane is prettier than Heather." The problems were given to 18 frontal patients. Goel and colleagues found that for the determinate problems, both valid and invalid (eg Michael is smarter than Mary), the left frontal group was worse than either healthy controls or the right frontal group. However, for the indeterminate problems, not well-structured, it was the right frontal group who were much worse than either of the other two groups, who did not differ..

Goel et al use the mental models approach of Johnson-Laird (1983) and hold that the indeterminate problems require the construction of at least two models for the alternative possibilities together with a representation that one or the other can be correct. They further argued that the left frontal lobe is adept at constructing determinate and unambiguous representations, whereas the right frontal lobe is needed to maintain "fluid, indeterminate, vague and ambiguous representations" (p. 2249). The study of Goel et al is rather small for strong theoretical conclusions, but as we will see its results resonate with other findings. 


\section{From lateral transformations to strategy shifts}

In an earlier paper, a single case study of an architect who had had a right frontal meningioma removed, Goel \& Grafman (2000) made a different if related contrast between the functions of the left and right PFC. Despite having an IQ of 125 and a maintained ability to carry out the basic skills of his profession, the patient was unable to operate effectively as an architect. Goel \& Grafman argued that he had retained the ability to make what they called "vertical transformations", namely more detailed versions of the same idea. What he had lost was held to be the ability to make "lateral transformations", where one moves from one idea to a different type of idea, which the authors held to be a function of the right frontal lobe.

Support for a related idea comes from a rather surprising source. In the attempt to develop a task requiring cognitive inhibition, Burgess \& Shallice (1996b) invented the Hayling Sentence Completion Test. In section B, subjects are presented with a sentence frame, such as The ship sank very close to the .... The task of the subject is to give a word unrelated to the completion of the sentence or to any word in the sentence. Banana would be an example. In an initial study of 91 patients, the Hayling B test proved to be highly sensitive to frontal lesions. Patients with anterior lesions produced more than double the error score of either posterior-lesioned patients or healthy controls. No significant lateralisation effects were found.

This looks like a difficulty in inhibition. However, it was noted that after a few trials, healthy controls tended to develop a strategy of looking round the room to select an object or of making an association with their previous response. Their aim was to produce a word before the sentence frame was presented. They then no longer had to inhibit the completion; they merely had to check that their already generated word did not by chance relate to the sentence frame. Anterior-lesioned patients gave far fewer responses that fitted either of these two 
strategies than did posterior-lesioned patients or healthy controls. They did not generate an effective strategy to circumvent the difficulty of the task.

Three studies indicated there are surprising right frontal involvements in this entirely verbal task. Roca et al (2010) were examining the extent to which fluid intelligence scores could explain frontal deficits in a number of tasks with 44 frontal patients. As discussed earlier, they found that for five tasks, one of which was a much shortened version of the Hayling test, the frontal deficit could not be explained as merely a consequence of impairment in fluid IQ. Six patients performed particularly badly on this set of tasks. Five of them had right frontal lesions.

In the second study using the full Hayling test, Volle et al (2012) tested 45 patients with focal cortical lesions. They then used two critical lesion localisation procedures. For both clinical measures of Hayling B, of reaction time and errors respectively, the critical lesion sites were in the right frontal lobe. For the more sensitive lesion localisation procedure, the reaction time slowing localised to right lateral areas 45 and 47, and increased errors to right orbitofrontal area 11.

Robinson et al (2015) gave the test to 90 focal frontal lesion patients, and used the StussAlexander grouping method. On the reaction time measure, it was the right lateral group that were grossly slow - worse than four times the healthy control group - while the left lateral group did not differ from controls. On the error measure, it was the right lateral group again that was the only patient group significantly worse than controls, making more than three times as high an error score. Moreover, they made very few responses indicating use of an effective strategy. More specifically, within the lateral frontal cortex, it was the inferior regions again where the difference lay between the effects of left and right lesions. 
More specifically, the performance on the Hayling test of right frontal patients with lateral and orbitofrontal lesions was compared on a new small sample. Right lateral patients were found to make many suppression errors, to produce very few strategy-connected words and to require longer 'thinking' times, known to correlate with fewer strategy responses. In contrast the orbitofrontal group performed normally. This supports the notion that it is the inferior right lateral cortex rather than the orbitofrontal cortex that is involved in strategy production (Cipolotti et al 2015c).

A general inhibition problem is an implausible explanation of the right lateral impairment. Cipolotti et al (2016) tested 30 frontal patients on both the Hayling task and the Stroop. The right frontal group was much worse than the left frontal one on the Hayling task, but for the Stroop there was an insignificant effect in the other direction.

By contrast, the notion that the right inferior lateral regions are critical for novel strategy production in problem-solving has been supported by two studies, one employing functional imaging and the other cortical thickness differences across normal subjects. Both studies used problem-solving tasks which involve an insightful lateral move to produce a novel strategy. One was Guilford's matchstick task (Goel \& Vartanian, 2005), the other the socalled Nim or Subtraction game (Seyed-Allaei, in press). Both found the critical area to be right area 47. Whether its role lies in the creation of a novel structure or plan or the realisation of the inadequacy of an earlier strategy remains to be established.

\section{Planning for future action}

Reasoning needs to be implemented in action, and often after a gap in time. Intentions need to be set up and realised later. Typically, other tasks have to be carried out in the interval. So, one has a multitasking situation. Shallice \& Burgess (1991) described three frontal patients who performed well on a wide range of clinical tests of frontal lobe function but were 
specifically impaired when given two tests of multitasking. Each of these tests - Six Elements and Multiple Errands - required patients to organise themselves to interleave a number of different tasks without cues as to when to switch, while obeying a set of simple rules written on a card in front of them. This study showed that multitasking was a separable frontal function. The one patient, AP, in whom the lesion could be well localised, had a bilateral lesion of frontopolar cortex (areas10 and 11) (Shallice \& Cooper, 2011). Burgess et al (2000) used another multitasking test, the Greenwich, which required three different tasks to be interleaved over 10 minutes. When memory was not impaired, poor overall performance was associated with lesions to the more polar and medial aspects of areas 8,9,10. Area 10 appears to be critical. Roca et al (2011) compared 7 frontal patients with area 10 damage to 8 without. They were more impaired in multitasking but less so on response inhibition and abstract reasoning.

That the temporal aspect of setting up and realising intentions may indeed be the core deficit of the multitasking impairment is shown by a study of Volle et al (2011). With the assistance of a stopwatch, 45 patients with focal lesions had to press a spacebar every 30 s while carrying out another task. The 8 patients with area 10 lesions pressed the spacebar once every 48s by comparison with every 32 s for the other patients. On control tasks not involving time, the area 10 patients were unimpaired.

Functional imaging studies, too, have given strong parallel evidence for the involvement of bilateral area 10 in multitasking, and in particular of the generation and realisation of intentions (Koechlin et al, 1999; Burgess et al, 2001, 2011).

\section{THEORETICAL CONCLUSIONS}


In this review we have focused on neuropsychological group studies of what we termed active thought and in the localisation of the principal processing components of a variety of tasks involving it. We have assumed that different localisations imply different computational functions. The most basic conclusion one draws from the neuropsychological literature is that the prefrontal cortex has a complex computational structure with a large set of subsystems combining to realise active thought. This is because impairments at the Supervisory level differ qualitatively on at least some combinations of lateral vs medial, left vs right, anterior vs posterior prefrontal and dorsal vs ventral.

In addition, most "frontal" tasks involve many components. Hence, the complexity of the neurocognitive architecture could well be greater than neuropsychological group studies alone currently indicate. This is because they pick out one or a very few critical regions. For instance, we have shown that right lateral frontal systems for novel strategy selection are important in carrying out the Hayling task. Yet Robinson et al (2016) have recently described two patients with different types of difficulty on the task, one clearly of inhibition. Both had left frontal lesions! The task undoubtedly involves multiple systems relevant for active thought.

In this case why are neuropsychological studies valuable? They clearly show the affected systems are crucial. In addition, though, they complement functional imaging findings informatively, both with respect to lateralisation of function and to the role of networks or their constituent subsystems. Regarding lateralization of functions, one frequently obtains the impression from the imaging literature that the two frontal cortices have basically equivalent functions; activation is often bilateral. The neuropsychological literature provides a different perspective. The two lateral prefrontal cortices appear to have markedly different functions with respect to active thought. 
There are a number of different ways in which these contrasting functions have been characterised. Thus, Stuss \& Alexander (2007) and Shallice \& Gillingham (2012) contrast task-setting, setting up a program (left), with active monitoring (right). The latter is well supported by the currently reviewed studies, the former by the Morris et al study of Tower of Hanoi. Goel and colleagues (2000, 2007), instead, make the contrast between vertical operations in a well-structured problem space (left) and lateral ones in an ill-structured space (right). This fits the results on deduction and the Tower-tasks (left) and the Hayling task well. Computationally, one can combine these two sets of contrasts. The left lateral region, becomes the site where Duncan's serially operating program is realised; this fits too with the task switching studies. The program then runs on systems in premotor and posterior cortices. By contrast, the right lateral region would be where processes operate in parallel either separately to detect any from a range of potential errors (active monitoring) or in combination to produce a novel strategy. This would fit with the left lateral region having a much higher degree of internal inhibition than the right, since at each stage it selects top-down one from a range of possible thought and action schemas.

Within the left lateral frontal lobe, the contrasting localisations of deduction (ventrolateral) and Tower-task operations (dorsolateral) fits roughly with a Petrides-like anatomical perspective. Cognitively, the contrast supports the view that rule-based mental logic and mental model-based reasoning both exist but rely on anatomically different systems (Goel, 2007). As far as mental model-based reasoning is concerned, Knauff (2013) has argued that the model itself is parietally located, and the existence of a qualitatively organised representation of objects in space in the right parietal lobe (Buiatti et al, 2011) supports this.

Regarding the contrast between neuropsychology and functional imaging on the role of networks or their constituent subsystems, imaging provides evidence on the network of 
systems involved in task execution. Focal lesion patients instead provide evidence ideally on single systems. From this perspective, Duncan \& Owen's fronto-parietal multiple demand network, may be seen as composed by a variety of special-purpose subsystems which combine to realise, for instance, mental model-based reasoning in tests of fluid IQ.

The clearest example of this functional distinction between parts of the network, is the contrast between lesions to lateral and superior medial frontal regions. Both contain parts of the multiple demand network. However, lesions affect the two regions differently across a range of neuropsychological tests, including reaction time, fluency and reasoning tasks. On the current approach the superior medial PFC energises supervisory operations, but the lateral PFC implements them; the two regions have different functions.

The impaired performance on different tasks of patients with lesions in the same region, can also give rise to theoretical questions. Consider the left inferior frontal region. We argued that it is involved in constructing the preverbal message, but also in the representation of abstraction. Both of these require hierarchically organised structures relating to language. But do they involve the same system? We will not know until it is investigated whether dissociations can exist between tasks involving them.

Neuropsychological findings on active thought do not just show that certain brain systems are critical for task-execution. In addition, they complement findings from functional imaging in two different ways. Rather than giving information on whole networks, they highlight the role of the systems of which they are composed. Secondly, rather than downplaying differential lateralisation of function, they emphasise it. Whether they can also help with the key question of how these supervisory systems interact remains to be seen.

\section{Summary Points}


1. For active thought processes, neuropsychology provides valuable evidence on underlying functional subsystems, and their lateralisation, rather than on whole networks.

2. For medial PFC the subsystems, to which such evidence relates, are critical for energising supervisory processes.

3. For left lateral PFC, they are critical for top-down schema activation, updating, deduction and more anteriorly, abstraction.

4. For left ventrolateral PFC, they help to construct preverbal messages.

5. For right lateral PFC, they underpin active monitoring and, more inferiorly, are critically involved in production of novel strategies.

6. For frontopolar PFC a key role is the setting-up and maintenance of intentions.

\section{Future Issues}

1. For models of fronto-parietal control networks, of which the multiple demand network is one, are the frontal components functionally different or functionally equivalent to the parietal components?

2. Does the left lateral PFC have stronger inhibition internal to the region than the right lateral PFC, as suggested above?

3. For some claimed processes (eg active monitoring) and even some tasks (eg Hayling B), there is a broad agreement across studies about which frontal lobe plays the more critical role, but there is disagreement over the specific parts of the lobe responsible. Is this due to variations across samples of patients tested or due to subtle differences in the cognitive processes employed to perform the particular version of the task used.

4. Abstraction and forming a preverbal message both involve more anterior parts of the inferior left lateral frontal lobe. Do they have any processes in common? For instance, extrapolating from Hagoort's (2013) ideas on localisation of so-called unification 
processes, could the region be required for the construction of multi=-level structures (Shallice \& Cooper, 2013).

5. Are impairments following lesions to the inferior lateral right frontal region in tasks like the stop task due to impairments to systems controlling response inhibition or to those controlling bottom-up attention?

6. What are the involvements of the right frontal region in novel strategy attainment tasks, such as Hayling B? Does it contain systems that create a novel structure or plan, or systems determining that the preceding strategy was inadequate and so needs changing, or are there yet further possibilities?

\section{ACKNOWLEDGMENTS}

We would like to thank Edgar Chan for his comments on an earlier version of the paper and Sara Gharooni for her assistance in preparing the paper. 


\section{LITERATURE CITED}

Aron AR, Fletcher PC, Bullmore ET, Sahakian BJ, Robbins TW. 2003. Stop-signal inhibition disrupted by damage to right inferior frontal gyrus in humans. Nat. Neurosci. 6(2):115-116

Aron AR, Monsell, S. Sahakian BJ, Robbins TW. 2004. A componential analysis of taskswitching deficits associated with lesions of left and right frontal cortex. Brain, 127(7):156173.

Bates E, Wilson SM, Saygin AP, Dick F, Sereno MI, et al. 2003. Voxel-based lesionsymptom mapping. Nat. Neurosci. 6(5):448-50

Burgess PW, Gonen-Yaacovi G, Volle E. 2011. Functional neuroimaging studies of prospective memory: what have we learned so far? Neuropsychologia 49(8):2246-57

Burgess PW, Quayle A, Frith CD. 2001. Brain regions involved in prospective memory as determined by positive emission tomography. Neuropsychologia 39(6):545-55

Burgess PW, Shallice T. 1996a Bizarre responses, rule detection and frontal lobe lesions. Cortex 32:241-59 
Burgess PW, Shallice T. 1996b. Response suppression, initiation and strategy use following frontal lobe lesions. Neuropsychologia 34(4):263-72

Burgess PW, Veitch E, de Lacy Costello A, Shallice T. 2000. The cognitive and neuroanatomical correlates of multitasking. Neuropsychologia 38(6):848-63

Buiatti T, Mussoni A, Toraldo A, Skrap M, Shallice T. 2011. Two qualitatively different impairments in making rotation operations. Cortex 47(2):166-79

Caramazza A. 1986. On drawing inferences about the structure of normal cognitive systems from the analysis of patterns of impaired performance: The case for single-patient studies. Brain Cogn. 5(1):41-66

Chan E, MacPherson SE, Robinson G, Turner M, Lecce F, et al. 2015. Limitations of the trail making test part-B in assessing frontal executive dysfunction. J. Int. Neuropsychol. Soc. 21(02):169-74

Chua EF, Schacter DL, Rand-Giovannetti E, \& Sperling RA 2006. Understanding metamemory: neural correlates of the cognitive process and subjective level of confidence in recognition memory. Neuroimage 29(4):1150-60.

Cipolotti L, Healy C, Chan E, Bolsover F, Lecce F, et al. 2015a. The impact of different aetiologies on the cognitive performance of frontal patients. Neuropsychologia 68:21-30 
Cipolotti L, Healy C, Chan E, MacPherson SE, White M, et al. 2015b. The effect of age on cognitive performance of frontal patients. Neuropsychologia 75:233-41

Cipolotti L, Healy C, Spanò B, Lecce F, Biondo F, et al. 2015c. Strategy and suppression impairments after right lateral prefrontal and orbito-frontal lesions. Brain 139(2):e10

Cipolotti L, Spanò B, Healy C, Tudor-Sfetea C, Chan E, et al. 2016. Inhibition processes are dissociable and lateralized in human prefrontal cortex. Neuropsychologia 93:1-12

Cooper R, Shallice T. 2000. Contention scheduling and the control of routine activities.

Cogn. Neuropsych. 17(4) 297-338

Costello AdL, Warrington EK. 1989. Dynamic aphasia: the selective impairment of verbal planning. Cortex 25(1):103-14

Crescentini C, Seyed-Allaei S, Vallesi A, Shallice T. 2012. Two networks involved in producing and realizing plans. Neuropsychologia 50(7):1521-35

Croquelois A, Bogousslavsky J. 2011. Stroke aphasia: 1,500 consecutive cases. Cerebrovasc. Dis. 31(4):392-99

Cummings JL. 1993. Frontal-subcortical circuits and human behavior. Arch. Neurol. 50(8): $873-880$ 
Delis DC, Kaplan E, Kramer JH. 2001. Delis-Kaplan executive function system (D-KEFS). San Antonio: Psychological Corporation

De Renzi E, Barbieri C. 1992. The incidence of the grasp reflex following hemispheric lesion and its relation to frontal damage. Brain 115(1):293-313

Derrfuss J, Brass M, Neumann J, von Cramon DY. 2005. Involvement of the inferior frontal junction in cognitive control: Meta-analyses of switching and Stroop studies. Hum. Brain Mapp. 25(1):22-34

D'Esposito M, Postle BR. 1999. The dependence of span and delayed-response performance on prefrontal cortex. Neuropsychologia 37:1303-15.

Duncan J. 2010. The multiple-demand (MD) system of the primate brain: mental programs for intelligent behaviour. Trends Cogn. Sci. 14(4):172-79

Duncan J, Owen AM. 2000. Common regions of the human frontal lobe recruited by diverse cognitive demands. Trends Neurosci. 23(10):475-83

Duncan J, Seitz RJ, Kolodny J, Bor D, Herzog H, et al. 2000. A neural basis for general intelligence. Science 289(5478):457-60

Fleck MS, Daselaar SM, Dobbins IG, Cabeza R. 2006. Role of prefrontal and anterior cingulate regions in decision-making processes shared by memory and nonmemory tasks. Cereb. Cortex 16(11):1623-30 
Gentner D, Goldin-Meadow S (Eds). 2003. Language in mind: Advances in the study of language and thought. Cambridge, Mass. MIT Press

Gilaie-Dotan S, Saygin AP, Lorenzi LJ, Rees G, Behrmann M. 2015. Ventral aspect of the visual form pathway is not critical for the perception of biological motion. P. Natl. Acad. Sci.USA 112(4): E361-E370

Goel V. 2007. Anatomy of deductive reasoning. Trends Cogn. Sci. 11:435-41

Goel V, Grafman J. 2000. Role of the right prefrontal cortex in ill-structured planning. Cogn. Neuropsych. 17(5): 415-436

Goel V, Tierney M, Sheesley L, Bartolo A, Vartanian O, Grafman J. 2007. Hemispheric specialization in human prefrontal cortex for resolving certain and uncertain inferences. Cereb. Cortex 17(10): 2245-50

Goel V, Vartanian O. 2005. Dissoicating the roles of right ventral lateral and dorsal lateral prefrontal cortex in generation and maintenance of hypotheses in set-shift problems. Cereb. Cortex 15:1170-77

Goldstein K. 1936. The significance of the frontal lobes for mental performances. J. Neurol. Psychopathol. 1:27-40

Hagoort P. 2013. MUC (memory, unification, control) and beyond. Front. Psychol. 4:416 
Hampshire A, Chamberlain SR, Monti MM, Duncan J, Owen AM. 2010. The role of the right inferior frontal gyrus: inhibition and attentional control. Neuroimage 50(3):1313-19

Henson RN, Greve A, Cooper E, Gregori, M, Simons JS, et al. 2016. The effects of hippocampal lesions on MRI measures of structural and functional connectivity.

Hippocampus 26(11):1447-63

Johnson-Laird PN. 1983. Mental models: Towards a cognitive science of language, inference, and consciousness. Cambridge, MA: Harvard University Press

Jung-Beeman M. 2005. Bilateral brain processes for comprehending natural language. Trends Cogn. Sci. 9(11): 512-18

Kahneman D, Frederick S. 2002. Representativeness revisited: Attribute substitution in intuitive judgment. In Heuristics of intuitive judgement: extensions and applications. Ed. T Gilovich, D Griffin, D Kahneman, pp49-81. New York: Cambridge University Press Kaller CP, Rahm B, Spreer J, Weiller C, Unterrainer JM. 2011. Dissociable contributions of left and right dorsolateral prefrontal cortex in planning. Cereb. Cortex 21(2):307-17

Karnath HO, Steinbach JP. 2011. Do brain tumours allow valid conclusions on the localisation of human brain functions?-Objections. Cortex 47(8):1004-06

Kerns JG, Cohen JD, MacDonald AW, Cho RY, Stenger VA, Carter CS. 2004. Anterior cingulate conflict monitoring and adjustments in control. Science 303(5660): 1023-26 
Khemlani S, Johnson-Laird PN. 2012. Theories of the syllogism: A meta-analysis. Psychol. Bull. 138(3):427-57

Knauff M. 2013. Space to reason: A spatial theory of human thought. Cambridge, MA: MIT Press

Koechlin E, Basso G, Pietrini P, Panzer S, Grafman J. 1999. The role of the anterior prefrontal cortex in human cognition . Nature 399:148 - 51

Levelt WJM. 1989. Speaking: From intention to articulation. Cambridge, MA:MIT Press

Lhermitte F. 1983. 'Utilization behaviour'and its relation to lesions of the frontal lobes. Brain 106(2): 237-55

Luria AR. 1966. Human brain and psychological processes. New York: Harper \& Row

Luria AR. 1970. Traumatic aphasia: Its syndromes, psychology and treatment. Mouton: The Hague

MacPherson SE, Healy C, Allerhand M, Spano B, Tudor-Sfetea C, White M, et al. In press. Cognitive reserve and cognitive performance of patients with focal frontal lesions. Neuropsychologia. 
MacPherson SE, Turner MS, Bozzali M, Cipolotti L, Shallice T. 2010. Frontal subregions mediating Elevator Counting task performance. Neuropsychologia 48(12):3679-82

Mah YH, Husain M, Rees G, Nachev P. 2014. Human brain lesion-deficit inference remapped. Brain 137(9):2522-31

Manly T, Robertson IH, Anderson V, Nimmo-Smith I. 1994. The Test of Everyday Attention (TEA-CH). Bury St. Edmunds: Thames Valley Test Company.

McDonald CR, Delis DC, Kramer JH, Tecoma ES, Iragui VJ. 2008. A componential analysis of proverb interpretation in patients with frontal lobe epilepsy and temporal lobe epilepsy: relationships with disease-related factors. Clin. Neuropsychol. 22(3):480-96

Miller EK, Cohen JD. 2001. An integrative theory of prefrontal cortex function. Annu. Rev. Neursci. 24(1):167-202

Milner B. 1963. Effects of different brain lesions on card sorting: The role of the frontal lobes. Arch. Neurol. 9(1):90-100

Miyake A, Friedman NP. 2012. The nature and organisation of individual differences in executive functions: four general conclusions. Curr. Dir. Psychol. Sci. 21(1):8-14

Morris RG, Miotto EC, Feigenbaum JD, Bullock P, Polkey CE. 1997. Planning ability after frontal and temporal lobe lesions in humans: The effects of selection equivocation and working memory load. Cogn. Neuropsych. 14(7):1007-27 
Murphy P, Shallice T, Robinson G, MacPherson SE, Turner M, et al. 2013. Impairments in proverb interpretation following focal frontal lobe lesions. Neuropsychologia 51(11):2075-86

Norman DA, Shallice T. 1986. Attention to Action. In Consciousness and self-regulation: Advances in research and theory. Ed. RJ Davidson, GE Schwartz, D Shapiro, pp.1-18. New York: Springer

Papagno C, Fogliata A, Catricala E, Miniussi C 2009. The lexical processing of abstract and concrete nouns. Brain Research 1263:7886

Papagno C, Miracapillo C, Casarotti A, Romero Lauro LJ, Castellano A. et al. 2011. What is the role of the uncinate fasciculus? Surgical removal and proper name retrieval. Brain 134(2):405-14

Paus T, Kalina M, Patočková L, Angerova Y, Cerny R, et al. 1991. Medial vs lateral frontal lobe lesions and differential impairment of central-gaze fixation maintenance in man. Brain, 114(5): 2051-67.

Petrides M. 1994. Frontal lobes and working memory: evidence from investigations of the effect of cortical excisions in nonhuman primates. In Handbook of Neuropsychology Vol. 9. Ed. F Boller, J Grafman, pp, 59-82. Amsterdam: Elsevier Science

Picton TW, Stuss DT, Alexander MP, Shallice T, Binns MA, Gillingham S. 2007. Effects of focal frontal lesions on response inhibition. Cereb. Cortex 17(4):826-38 
Posner MI, DiGirolamo GJ. 1998. Conflict, target detection and cognitive control. In. The Attentive Brain. Ed. R Parasuraman, pp. 401-423. Cambridge, MA: MIT Press

Rapp AM, Leube DT, Erb M, Grodd, Kircher TT. 2004. Neural correlates of metaphor processing. Cogn. Brain Res. 20(3):395-402

Reverberi C, Cherubini P, Frackowiak RS, Caltagirone C, Paulesu E, Macaluso E. 2010. Conditional and syllogistic deductive tasks dissociate functionally during premise integration. Human brain mapping 31(9):1430-45

Reverberi C, Lavaroni A, Gigli GL, Skrap M, Shallice T 2005. Specific impairments of rule induction in different frontal lobe subgroups. Neuropsychologia 43:460-72

Reverberi C, Shallice T, D’Agostini S, Skrap M, Bonatti LL. 2009. Cortical bases of elementary deductive reasoning: Inference, memory, and metadeduction. Neuropsychologia 47(4):1107-16

Rizzo A, Ferrante D, Bagnara S. 1995. Handling human error. Expertise and technology: Cognition and human-computer cooperation, ed. PC Hoc, E Cacciabue, pp. 195-212. Hillsdale, NJ: Lawrence Erlbaum

Robinson G, Blair J, Cipolotti L. 1998. Dynamic aphasia: an inability to select between competing verbal responses? Brain 121(1)77-89 
Robinson G, Cipolotti L, Walker DG, Biggs V, Bozzali M, Shallice T. 2015. Verbal suppression and strategy use: a role for the right lateral prefrontal cortex? Brain, 138(4):1084-96

Robinson G, Shallice T, Cipolotti L. 2005. A failure of high level verbal response selection in progressive dynamic aphasia. Cognitive Neuropsych. 22(6):661-94

Robinson G, Shallice T, Bozzali M, Cipolotti L. 2010. Conceptual proposition selection and the LIFG: neuropsychological evidence from a focal frontal group. Neuropsychologia 48(6):1652-63

Robinson G, Shallice T, Bozzali M, Cipolotti L. 2012. The differing roles of the frontal cortex in fluency tests. Brain 135(7):2202-14

Robinson G, Walker DG, Biggs V, Shallice T. 2016. When does a strategy intervention overcome a failure of inhibition? Evidence from two left frontal brain tumour cases. Cortex 79:123-9

Roca M, Parr A, Thompson R, Woolgar A, Torralva T, et al. 2010. Executive function and fluid intelligence after frontal lobe lesions. Brain 133:234-47.

Roca M, Torralva T, Gleichgerrcht E, Woolgar A, Thompson R, et al. 2011. The role of area 10 (BA10) in human multitasking and in social cognition: a lesion study. Neuropsychologia, 49(13):3525-31 
Rorden C, Karnath HO. 2004. Using human brain lesions to infer function: a relic from a past era in the fMRI age? Nat. Rev. Neurosci. 5(10):812-19

Szczepanski SM, Knight RT 2014. Insights into human behavior from lesions to the prefrontal cortex. Neuron, 83(5):1002-18.

Seyed-Allaei S, Avanki ZN, Bahrami B, Shallice T. in press. Major thought restructuring: The roles of the different prefrontal cortical regions. J. Cogn. Neurosci.

Shallice T. 1982. Specific impairments of planning. Philos. Trans. R. Soc. B 298(1089):199209

Shallice T. 2015. Cognitive neuropsychology and its vicissitudes: The fate of Caramazza's axioms. Cogn. Neuropsychol. 32(7-8):385-411

Shallice T, Burgess PW, Schon F, Baxter DM. 1989. The origins of utilization behaviour. Brain 112(6):1587-98

Shallice T, Burgess PW. 1991. Deficits in strategy application following frontal lobe damage in man. Brain 114(2):727-41

Shallice T, Cooper RP. 2011. The organisation of mind. Oxford: Oxford University Press

Shallice T, Cooper RP. 2013. Is there a semantic system for abstract words? Front. Hum. Neurosci. 7:175 
Shallice T, Gillingham SM. 2012. On neuropsychological studies of prefrontal cortex: The ROBBIA approach. In Principles of frontal lobe function. ed. DT Stuss, RT Knight, pp.475489. Oxford: Oxford University Press

Shallice T, Stuss DT, Picton TW, Alexander MP, Gillingham S. 2008. Multiple effects of prefrontal lesions on task-switching. Front. Hum. Neurosci. 2:2

Sharp DJ, Scott SK, \& Wise RJ 2004. Monitoring and the controlled processing of meaning: distinct prefrontal systems. Cereb. Cortex, 14(1):1-10

Stanovich, K.E., 1999. Who is rational?: Studies of individual differences in reasoning. Routledge: Psychology Press

Stuss DT, Alexander MP. 2007. Is there a dysexecutive syndrome? Philos. Trans. R. Soc. B 362(1481): 901-15.

Stuss DT, Alexander MP, Hamer L, Palumbo C, Dempster R, et al. 1998. The effects of focal anterior and posterior brain lesions on verbal fluency. J. Int. Neuropsych. Soc. 4(3):265-78

Stuss DT, Alexander MP, Shallice T, Picton TW, Binns MA, et al. 2005. Multiple frontal systems controlling response speed. Neuropsychologia 43(3)396-417

Stuss DT, Shallice T, Alexander MP, Picton TW. 1995. A multidisciplinary approach to anterior attentional functions. Ann. NY. Acad. Sci. 769(1):191-212 
Thompson-Schill SL, Swick D, Farah MJ, D’Esposito M, Kan IP, Knight RT. 1998. Verb generation in patients with focal frontal lesions: A neuropsychological test of neuroimaging findings. P. Natl. Acad. Sci. USA 95(26):15855-60

Tsuchida A, Fellows LK. 2013. Are core component processes of executive function dissociable within the frontal lobes? Evidence from humans with focal prefrontal damage. Cortex 49(7):1790-800

Urbanski M, Bréchemier ML, Garcin B, Bendetowicz D, de Schotten MT, et al. 2016. Reasoning by analogy requires the left frontal pole: lesion-deficit mapping and clinical implications. Brain 139(6):1783-99

Vallesi A, Mussoni A, Mondani M, Budai R, Skrap M, Shallice T. 2007b. The neural basis of temporal preparation: insights from brain tumor patients. Neuropsychologia 45(12):2755-63

Vallesi A, Shallice T, Walsh V. 2007a. Role of the prefrontal cortex in the foreperiod effect: TMS evidence for dual mechanisms in temporal preparation. Cerebral Cortex 17(2):466-74

Varley R. 2014. Reason without much language. Lang. Sci 46:232-44

Varley RA, Klessinger NJ, Romanowski CA, Siegal M. 2005. Agrammatic but numerate. $P$. Natl. Acad. Sci. USA 102(9): 3519-24. 
Volle E, de Lacy Costello A, Coates LM, McGuire C, Towgood K, et al. 2012. Dissociation between verbal response initiation and suppression after prefrontal lesions. Cereb.

Cortex 22:2428-40

Volle E, Gonen-Yaacova G, de Lacy Costello A, Gilbert SJ, Burgess PW. 2011. The role of rostral prefrontal cortex in prospective memory: a voxel-based lesion study.

Neuropsychologia 49(8):2185-98

Woolgar A, Parr A, Cusack R, Thompson R, Nimmo-Smith I, et al. 2010. Fluid intelligence loss linked to restricted regions of damage within frontal and parietal cortex. P. Natl. Acad. Sci. USA 107(33):14899-902

Yokoyama O, Miura N, Watanabe J, Takemoto A, Uchida S, et al. 2010. Right frontopolar cortex activity correlates with reliability of retrospective rating of confidence in short-term recognition memory performance. Neurosci. Res. 68(3):199-206 